Disponível em

http://www.anpad.org.br/rac

RAC, Curitiba, v. 14, n. 4, art. 5,

pp. 651-669, Jul./Ago. 2010

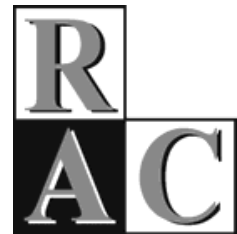

\title{
A Produção Científica Sobre Cultura Organizacional em Organizações Públicas no Período de 1997 a 2007: um Convite à Reflexão
}

\section{Scientific Production on Organizational Culture in Public Organizations from 1997 to 2007: an Invitation to Rethink and Reconsider}

\footnotetext{
* Endereço: Lindomar Pinto da Silva

Rua Adenil Falcão, 1823, Bairro Brasília, Feira de Santana/BA, 44088-270. Email: lpsilva@sefaz.ba.gov.br
}

Copyright (C) 2010 RAC. Todos os direitos, inclusive de tradução, são reservados. É permitido citar parte de artigos sem autorização prévia desde que seja identificada a fonte. 


\title{
RESUMO
}

Este trabalho analisa a produção científica sobre cultura organizacional em organizações públicas no Brasil, no período de 1997 a 2007. A pesquisa privilegiou os periódicos brasileiros mais conhecidos na comunidade acadêmica e os encontros promovidos pela ANPAD neste período. A produção foi analisada a partir dos aspectos relativos à continuidade da pesquisa em cultura organizacional em organizações públicas, características das pesquisas, métodos de pesquisa, instrumentos de medida, modelo de tratamento dos dados, teorias de base, vertente teórica e o alinhamento dos pesquisadores com o tema analisado. Os resultados mostram que a produção nesta temática é baixa, considerando que foram encontrados apenas 47 trabalhos publicados no período estudado. Indicam, ainda, que não há continuidade na produção de pesquisa voltada especificamente para os estudos culturais em organizações públicas, pois os autores, na sua maioria, publicaram apenas um trabalho neste período sobre este tema.

Palavras-chave: cultura organizacional; administração pública; produção científica.

\begin{abstract}
This work analyses the scientific production on Organizational Culture in public organizations in Brazil form 1997 to 2007. The research was based on Brazilian publications, such as well-known scientific magazines, and congresses promoted by ANPAD (Brazilian Association of Graduate Programs in Administration) during this period. The scientific production was analyzed regarding the continuation of the research in organizational culture in the Public Sector, measurement methods, data treatment models, the kind of theories used, the directions of such theories and the alignment of the researchers with the proposed theme. The results show that the production in the period studied was small, considering that we found only 47 studies published in the period mentioned. They also indicate that there is no continuity of production in the field and that the authors, in their majority, published just one work in 10 years on this theme.
\end{abstract}

Key words: organizational culture; public administration; scientific production. 


\section{INTRODUÇÃO}

O objetivo central deste trabalho é analisar a produção científica brasileira sobre cultura organizacional em organizações públicas. A pesquisa privilegiou os periódicos brasileiros mais conhecidos na comunidade acadêmica e os encontros promovidos pela ANPAD neste período.

O destaque feito para as organizações que integram o setor público decorre da convicção de que existem especificidades culturais nessas organizações que as distingue das empresas privadas e, que, portanto, dariam direcionamentos e achados diferentes às pesquisas nelas realizadas. Outro elemento motivador deste estudo, focado em cultura organizacional em organizações públicas, foi o entendimento de que cultura pode ser fator determinante no desempenho dessas organizações, sobretudo considerando-se as medidas adotadas a partir da Reforma do Estado de 1995, as quais pressupunham intervenções nas dimensões institucional-legal, cultural e na dimensão-gestão. A dimensão cultural destaca-se no Plano Diretor de Reforma do Aparelho do Estado, como elemento necessário e como dimensão central a ser transformada. Fortemente baseada em valores burocráticos, segundo o plano, esta cultura organizacional deveria submeter-se a mudanças que fossem capazes de transformá-la em uma cultura gerencial (Ministério da Administração Federal e da Reforma do Estado [MARE], 1995).

Reforma e mudança no setor público brasileiro não são fenômenos novos. No entanto, a Reforma do Estado implementada na década de 90, formalizada no Plano Diretor da Reforma do Aparelho do Estado [PDRAE], ganhou centralidade na agenda política e no discurso da comunidade acadêmica, não apenas pela sua abrangência ou por ser a mais recente, mas, sobretudo, por trazer, em seu bojo, propostas de transformações estruturais no papel desempenhado pelo Estado e na sua relação com a sociedade. Essa redefinição de espaços e papéis do Estado implicou, também, uma transformação no modo de administrar as organizações públicas. Um dos pilares de sustentação dessas mudanças é o administrador público para o qual se deveria transferir maior autonomia e atribuir maior responsabilidade na gestão dos recursos públicos. A opção deste estudo em ressaltar o processo de reforma iniciado em 1995 deve-se justamente à ênfase na busca de transformação da cultura do setor público e do destaque dado ao servidor público como elemento essencial para a implementação de qualquer processo de transformação organizacional no setor público.

Neste cenário que apresenta a cultura organizacional como elemento essencial para o sucesso da Reforma do Estado implementada a partir de 1995, este trabalho inicia partindo do pressuposto da existência de uma produção científica vasta e diversificada sobre esta temática, capaz de demonstrar mudanças significativas ocorridas, com repercussões no desempenho das organizações públicas, como preconizado pela reforma. Em outras palavras, diante deste quadro de exigência de mudança cultural parece natural que, na medida em que processos de mudança em organizações governamentais seguissem seu curso, pesquisas científicas fossem realizadas no intuito de avaliar os impactos dos diversos programas na efetivação da mudança cultural preconizada pela reforma.

Partindo desse entendimento, este trabalho buscou verificar o desenvolvimento e o direcionamento das pesquisas sobre cultura organizacional em organizações públicas no Brasil, no período pósreforma do Estado, de 1997 a 2007. Procurou identificar as principais características desses estudos relativas à sua continuidade e sistematização, métodos de pesquisa, instrumentos de medida, modelo de tratamento dos dados, teorias de base, vertente teórica e o alinhamento dos pesquisadores com o tema analisado, destacando, ainda, o perfil dos autores e o campo de estudos ao qual se filiam. $\mathrm{O}$ estudo inicia com breve discussão dos principais conceitos de cultura organizacional, resgata e contextualiza a trajetória de formação da administração pública brasileira, apresenta a metodologia utilizada no desenvolvimento da pesquisa para, por fim, sistematizar e discutir os dados coletados. 


\section{CULTURA ORGANIZACIONAL - BASES TEÓRICAS}

A cultura organizacional constitui elemento importante no contexto organizacional, o que leva diversos autores a estudarem esta temática de modo contínuo e sistemático. Desde a década de 80, quando surgiram os primeiros estudos sobre cultura organizacional, foi-lhe atribuída a capacidade de solucionar todos os problemas organizacionais. Inúmeros estudos vêm sendo realizados como forma de compreender os aspectos mais importantes deste construto, que se revela cada dia mais interessante, do ponto de vista da pesquisa científica.

Apesar da importância atribuída à cultura organizacional para as organizações, esta vem sendo conceituada de várias maneiras: (a) para Schein (2004, p. 17) ela é "o conjunto de pressupostos básicos que um grupo inventou, descobriu ou desenvolveu que funcionaram bem o suficiente para serem considerados válidos e ensinados a novos membros, como a forma correta de perceber, pensar e sentir" (Schein, 2004, p. 17); (b) para Aktouf (1994, p. 42), a cultura é "uma maneira diferente de designar um sistema informal generalizado, irracional e talvez, profundamente subjetivo ou inconsciente”.

Para fins deste estudo, considera-se que o conceito de cultura organizacional deve abranger um conjunto de valores que refletem e se refletem em artefatos visíveis, que se alteram em função de fatores internos e/ou externos à própria cultura organizacional. A cultura deve considerar as diversas interações de indivíduos e grupos, o que lhe confere características específicas dentro do mesmo grupo organizacional em função de fatores diversos, como o grupo profissional no qual se situam, os interesses individuais e do grupo, etnia e classe social. Os artefatos visíveis ou elementos materiais da cultura são aqueles que podem ser vistos, sentidos e ouvidos. Incluem-se aí produtos, estrutura física, linguagem, tecnologia, roupas, mitos, histórias, lista de valores publicados, rituais e cerimônias. Schein (2004) considera que estes elementos são de fácil visualização, mas de difícil interpretação. Esta percepção de Schein (2004) pode ser reforçada tanto pela antropologia, em Sahlins (1997), como na teoria das organizações através de Mascarenhas (2007); Meyerson e Martin (1987); Freitas (2007); Fleury (1996).

Ao se referirem a um dos componentes dos artefatos, os rituais e as cerimônias, Deal e Kennedy (2000) entendem serem estes importantes não só para a identificação de uma cultura forte, mas também para a sua preservação. Nesta direção, Deal e Kennedy (2000, p. 63) consideram: "sem eventos expressivos qualquer cultura morre. Na ausência de cerimônias ou rituais, importantes valores não têm impacto algum”. Desse modo, os rituais e cerimônias, bem como os demais elementos componentes dos artefatos possuem a função de preservar os valores necessários à manutenção da organização, pois esses valores são os orientadores do comportamento dos indivíduos dentro das organizações. De fato, segundo Deal e Kennedy (2000, p.21), valor é o "pano de fundo de qualquer cultura corporativa. Como a essência da filosofia da companhia para a realização do sucesso, os valores servem de senso de direção comum para todos os empregados e guia de comportamento para o seu dia-a-dia”. Percebe-se que, na visão destes autores, da importância de uma compreensão clara dos valores e de sua internalização e prática por todos os funcionários, depende o sucesso organizacional. Contudo vale ressaltar que, em outras abordagens teóricas, a importância dos valores não está na instrumentalidade que possuem para o sucesso organizacional (Carrieri \& Leite-de-Silva, 2006; Freitas, 2007).

Os estudos de cultura organizacional, do ponto de vista teórico, podem ser direcionados e desenvolvidos por diversas abordagens e métodos. Há trabalhos quantitativos que abordam elementos da cultura organizacional nos quais, segundo estes autores, é possível quantificar os elementos da cultura organizacional (Deal \& Kennedy, 2000; Fleury, 1996; Hofstede, 2003; Schein, 2004). Há, também, trabalhos de cunho qualitativo, que abordam aspectos mais descritivos da cultura organizacional (Carrieri \& Leite-de-Silva, 2006; Cavedon \& Fachin, 2002; Freitas, 1991, 2007; Martin \& Frost, 2001; Mascarenhas, 2007; Meyerson \& Martin, 1987). 
Smircich (1983) considerou que há autores que percebem a cultura como dependente do ambiente e que a organização não tem controle sobre ela; considera, também, que há autores que entendem que a cultura é uma variável perfeitamente controlável dentro da organização, o que permite planejamentos sistemáticos do modelo de cultura mais adequado para a organização (Deal \& Kennedy, 2000; Schein, 2004). Há, entretanto, aqueles autores que acreditam na mudança cultural, mas desacreditam a capacidade de os gestores organizacionais atuarem sobre estas mudanças de forma tão efetiva quanto pensam os autores da corrente gerencialista/funcionalista. Na abordagem das limitações da ação dos gestores sobre a cultura destacam-se Sahlins (1985, 1997), Laraia (2001), Martin e Frost (2001), Meyerson e Martin (1987), Jaime e Serva (1995) e Jaime (2002).

Uma abordagem importante é destacada por Martin e Frost (2001) e Meyerson e Martin (1987). Para estes autores, as organizações podem ser pensadas em termos de culturas únicas, monolíticas e integradas. Podem ser pensadas como possuindo culturas diferenciadas que variam, em função dos diversos grupos dentro da organização. E também a cultura da organização pode ser pensada como completamente ambígua e paradoxal. Esta abordagem constitui instrumento de análise cultural importante na medida em que considera a cultura organizacional a partir de diversos atores e grupos de atores, que possuem sentidos e interesses diferentes sobre os mesmos elementos da organização (Mascarenhas, 2007; Sahlins, 1997). A organização deixa de ser vista como unidade totalmente integrada e passa a ser vista como conjunto de indivíduos que, em algum aspecto, podem ter valores totalmente compartilhados entre todos; em outros momentos, alguns valores só conseguem ser compartilhados por alguns indivíduos e alguns grupos, enquanto outros valores apenas são compartilhados temporariamente.

\section{A Administração Pública Brasileira: Ambiente Organizacional e Modelos de Gestão}

A administração pública brasileira foi sendo historicamente construída e consolidada ao longo de várias décadas, em uma trajetória marcada por movimentos reformistas que

se localizam tanto em alguns momentos episódicos das crises políticas e institucionais em que o país esteve envolvido, como nos períodos em que o próprio Estado brasileiro passou por momentos de crise que exigiram um redimensionamento e revisão de suas políticas e estratégias de desenvolvimento (Fadul \& Souza, 2005, p. 1).

No intuito de contextualizar a administração pública situando-a como campo de estudo, para aí localizar os estudos sobre cultura organizacional em organizações públicas, objeto deste artigo, resgatam-se alguns aspectos desta formação, associando-os às iniciativas de reforma.

Há praticamente consenso entre os estudiosos de que os anos 30, a partir do Estado Novo e da consolidação no Brasil de uma política de expansão do setor público, marcaram o início de uma administração pública brasileira constituída em moldes profissionalizados, inspirada no modelo de administração científica taylorista/fayolista, com uma gestão profissional e impessoal, aproximando-se de uma burocracia nos padrões weberianos. O movimento reformista ocorrido na década de 30; o fato que sobressai é a criação do Departamento Administrativo do Serviço Público [DASP], principal encarregado de viabilizar e canalizar os esforços de modernização do Estado e das organizações públicas no país é marcante na história da administração pública brasileira. Um dos grandes destaques da atuação do DASP refere-se à admissão por concurso e à inclusão de sistema mérito no serviço público.

A partir desta década ocorreu uma sucessão de reformas cujos processos, cada um a seu tempo, tiveram impactos mais ou menos profundos e moldaram a cultura das organizações da administração públicas, definindo, assim, as suas especificidades. Fadul e Souza (2005) classificam essas mudanças em duas grandes categorias, quais sejam: reformas paradigmáticas e reformas não paradigmáticas, colocando entre as primeiras aquelas mais abrangentes e significativas em relação ao seu impacto na estrutura administrativa do país, e entre as segundas, as que provocaram transformações menos amplas no setor público. 
Além da reforma da década de 30, entre as reformas paradigmáticas, inclui-se a ocorrida durante o regime militar de 1964, com a publicação do Decreto-Lei no 200 (1967), que já procurava delinear um modelo de administração pública gerencial, apontando a direção da adoção, pelo setor público, de um modelo gerencial oriundo da iniciativa privada. Nessa época estimulou-se a criação de empresas estatais e a expansão do sistema de mérito.

As mudanças empreendidas pela Constituição Federal de 88 podem ser reunidas no terceiro processo de reforma paradigmática. Esta Constituição enfatiza o processo de descentralização de políticas públicas e sociais, propiciando à administração pública um viés mais democrático, transparente, com maior controle social.

A quarta reforma foi a dos anos 90, iniciada no Governo Collor e consolidada de forma extensiva e aprofundada nos dois governos de Fernando Henrique Cardoso, com a construção do Plano Diretor da Reforma do Aparelho do Estado em 1995. Este plano propunha a adoção de um modelo de administração pública gerencial. Entre as reformas não paradigmáticas estão as que ocorreram no segundo governo Vargas; a reforma administrativa do governo Juscelino Kubitscheck (1956-1960); a reforma do Governo João Goulart (1962); e as mudanças realizadas no período do governo Collor de Melo (Fadul \& Souza, 2005).

Desta rápida e sucinta visão histórica da formação do contexto institucional no qual se inserem e atuam as organizações públicas brasileiras, percebem-se traços marcantes que essas reformas imprimiram nos modelos de gestão e, em conseqüência, na própria cultura organizacional do setor público que serão destacados a seguir. O movimento reformista ocorrido na década de 30 , com a criação do DASP, traz na sua base a adoção dos princípios de administração, consagrados cientificamente como modelo prescritivo da então teoria administrativa, com foco em as áreas de administração de pessoal, com a definição de critérios profissionais para ingresso no serviço público, desenvolvimento de carreiras e estabelecimento de regras de promoção baseadas no mérito (Marini, 1996, p. 17). Criando-se regras legais, formas de acesso aos cargos públicos e normas de condutas para os administradores públicos, estes estariam fora do campo de influência dos políticos, vistos como interessados em beneficiar-se da função pública. Desse modo, a reforma da década de 30 constrói e consolida o modelo burocrático brasileiro, buscando a separação entre política e administração, procurando romper com a cultura patrimonialista, sendo o patrimonialismo entendido como o uso privado daquilo que é público, ou seja, a apropriação da res publica do aparelho do Estado e do próprio Estado por grupos de interesse, com prática de concessão de benefícios governamentais para determinada clientela ou grupo político, em troca de favores, então vigente, herdada da colonização portuguesa.

Bresser Pereira (1998) defende que, na emergência do capitalismo e da democracia, era necessário não somente separar o público do privado, comum no patrimonialismo, mas também efetuar uma separação entre o político e o administrador público. A separação entre políticos e administradores, entretanto, não é vista como a solução dos problemas apontados pelo patrimonialismo. Ainda que uma distância seja necessária, a ação política permeia todos os níveis de atividade dentro do setor público. Não é sem causa que Pires e Macedo (2006) acreditam que uma das habilidades necessárias ao administrador público é a diplomacia como forma de não provocar divergências nas relações que são permeadas de estimas e jogos de poder, que se constituem nos verdadeiros indicadores de poder no Brasil. Além disso, Loureiro, Abrúcio e Rosa (1998) refutam esta segregação entre política e administração na medida em que é a capacidade de negociar do Poder Executivo ou do Legislativo e Judiciário, que permite ao administrador público executar as suas atividades, planejando e implementando as políticas públicas nacionais. Entretanto há que considerar que, naquele momento, a busca da separação se tornava imprescindível, na medida em que o rompimento com o patrimonialismo se fazia necessário, sob pena de inviabilização dos esforços de modernização por que passava o Estado brasileiro.

Já o Decreto-Lei $n^{0} 200$ (1967), além das iniciativas de reestruturação administrativa em diversos setores da administração federal, traz consigo importante inovação. Trata-se da introdução de valores gerenciais na administração pública, e desenvolve nas mais diversas organizações da administração 
indireta uma cultura gerencial semelhante ao modelo oriundo do setor privado da economia. Por sua vez, a descentralização político-institucional, trazida pela Constituição de 88, favoreceu a retomada da discussão da relação Estado e sociedade, historicamente autoritária, abrindo canais de diálogo e participação no processo de formulação de políticas públicas, com maior transparência na gestão, com impactos na cultura das organizações públicas.

Por fim, o marco histórico que motiva a elaboração deste artigo, a reforma implementada a partir de 1995 através do PDRAE, também chamada de Reforma Gerencial, tinha a intenção de superar os vícios da administração pública burocrática. Procura introduzir na administração pública brasileira um modelo de gestão com foco na eficiência, na busca de resultados e na busca de qualidade da prestação de serviços ao cidadão.

Uma análise do Plano Diretor da Reforma do aparelho do Estado brasileiro revela a importância dada ao que foi designado como cultura gerencial. O Estado devia efetuar os esforços necessários para criar e manter uma cultura gerencial capaz de apoiar as mudanças implementadas. Por isso o Plano Diretor reforça que "a implantação da nova cultura gerencial, centrada em resultados, na competição administrada e baseada na avaliação do desempenho, permitirão mudanças mais estruturais na direção pretendida” (MARE, 1995, p. 57). A cultura organizacional passa a ser tratada como o grande impulsionador da mudança gerencial.

Apesar da associação desses modelos de gestão - patrimonialista, burocrático e o gerencial - aos processos de reforma da administração pública brasileira que ocorreram no país, isso não significa dizer que esses modelos se sucederam na história, sendo um abandonado ou superado na medida em que o outro se instalava. É possível perceber, em organizações públicas brasileiras, mesmo as mais recentes e inovadoras, como as agências reguladoras, traços patrimonialistas, burocráticos e gerenciais presentes nos seus modelos de gestão.

Os modelos de gestão aqui apresentados significam, cada um no seu tempo, um conjunto de práticas políticas e de culturas organizacionais que prevaleceram num dado momento histórico da administração pública brasileira; suas evidências podem ser analisadas à luz das práticas administrativas pelas quais os negócios públicos são conduzidos. Esta associação, utilizando-se de períodos históricos, tem a finalidade específica de contextualizar e destacar os elementos da cultura organizacional que moldaram a administração pública brasileira ao longo de sua história.

\section{METODOLOGIA}

Este trabalho analisa as pesquisas produzidas sobre a temática da cultura organizacional em organizações públicas no Brasil, no período de 1997 a 2007, caracterizando-se como pesquisa quantitativa, de caráter exploratório. Inicialmente foram identificados intencionalmente nove periódicos editados no país e cinco anais de congressos realizados no Brasil, considerados importantes e representativos da área, conforme tabela 1 - Produção por periódico/anais de 1997 a 2007. A partir desse levantamento, foram selecionados, inicialmente pelos títulos, os diversos artigos publicados nos periódicos e anais que tratassem do tema da cultura organizacional em administração pública. A primeira dificuldade encontrada na realização desta seleção foi a identificação, através dos títulos dos trabalhos, de que eles tratavam de cultura organizacional em organizações públicas. Nem todos os títulos representam o menor resumo do conteúdo do artigo e muitos não trazem indicações do tema de que estão tratando. Avançar nesta etapa da pesquisa implicou necessariamente a leitura de diversos artigos, de modo a identificar o seu conteúdo exato.

Nesta primeira fase da pesquisa foram identificados 440 artigos que tratavam de cultura organizacional e a partir desta etapa foram selecionados 80 artigos que tratavam da temática da cultura organizacional, mencionando organizações públicas. Da leitura desses 80 artigos inicialmente selecionados percebeu-se que nem sempre tratavam dos conceitos teóricos discutidos pela cultura 
organizacional, e mais, de cultura organizacional na administração pública. Assim, desses artigos foram selecionados 47 para uma leitura mais detalhada e sistematizada, buscando identificar os elementos necessários para dar suporte a esta pesquisa.

Esses elementos, ou conjunto de dados utilizados no processo de coleta de dados para posterior análise, basearam-se em Camargos, Coutinho e Amaral (2005) e Sampaio e Perin (2006). São os seguintes: autores, quantidade de artigos por unidade da federação, quantidade de artigos por região, titulação dos autores, linha de pesquisa dos autores, continuidade das pesquisas na área de cultura organizacional em organizações públicas, produção total por ano, natureza da pesquisa, tipo de pesquisa, justificativa, teoria de base, objetivo e questão de pesquisa, hipótese e pressuposto, modelo da pesquisa, tipo de dados, mix de métodos, descrição dos procedimentos metodológicos, operação de variáveis, tipo de dados, tipo de amostra, instrumentos de coleta de dados, tamanho da população, tamanho da amostra, nível hierárquico dos entrevistados, tratamento estatístico dos dados, confrontação com a teoria de base, limitação do estudo, recomendação de pesquisa, recomendações aplicadas, validade externa, validade nomológica, vertente teórica, unidade cultural, valores culturais anteriores identificados, novos valores identificados, novos artefatos identificados e valores assumidos como modificados em função da implantação de novos artefatos. Esses dados foram tratados por estatística descritiva.

A outra parte da pesquisa tratou da origem e das publicações dos autores. Nesta parte, alinhada com a possibilidade de efetivamente contribuir para a construção de uma comunidade de discurso de pesquisa em cultura organizacional nas organizações públicas, buscou-se analisar o perfil dos autores. Essas informações foram buscadas nos currículos lattes de cada autor, de modo a identificar as suas linhas de pesquisas. Consistiu em analisar os títulos dos trabalhos desses autores, bem como outras informações disponibilizadas no currículo lattes para classificar os autores como pertencentes ou não à linha de pesquisa em organizações públicas e cultura organizacional.

\section{O que mostram os dados}

\section{a) Os Periódicos e Anais}

A primeira constatação que a análise revela é a baixa concentração de artigos sobre o tema nos periódicos mais direcionados à divulgação de pesquisas na própria área da administração pública. Nos Cadernos EBAPE.BR, por exemplo, neste período, não há publicação de artigo algum que abordam diretamente a temática. Em contraponto, os anais dos EnANPADs são os que apresentam a maior concentração de publicações sobre o tema, conforme Tabela 1, a seguir.

Tabela 1

\section{Produção por Periódico/Anais no Período de 1997 a 2007}

\begin{tabular}{lc}
\hline \multicolumn{1}{c}{ Periódico } & Total no Período \\
\hline Revista de Administração da USP & 1 \\
Revista de Administração de Empresas Eletrônica & 1 \\
Revista Organização \& Sociedade & 2 \\
Revista de Administração Pública & 6 \\
Revista de Administração Contemporânea & 6 \\
Revista de Administração Contemporânea eletrônica - RAC-@; & 0 \\
Brazilian Administration Review - BAR & 0 \\
Cadernos EBAPE.BR & 0 \\
Revista de Administração de Empresas - RAE & 0 \\
\hline
\end{tabular}

Continua 
A Produção Científica Sobre Cultura Organizacional em Organizações Públicas no Período de 1997 a 2007: 659 Um Convite à Reflexão

\section{Tabela 1 (continuação)}

\begin{tabular}{lc}
\hline \multicolumn{1}{c}{ Periódico } & Total no Período \\
\hline Anais EnANPAD & 16 \\
Anais Encontro de Estudos Organizacionais & 9 \\
Anais Encontro de Estratégia & 1 \\
Anais Encontro de Gestão de Pessoas e Relações de Trabalho & 3 \\
Anais Encontro de Administração Pública e Governança & 2 \\
TOTAL & 47 \\
\hline
\end{tabular}

Nota. Fontes: elaboração dos autores

\section{b) Os Autores}

Os autores que publicaram neste período totalizam apenas 79, com uma média de 1,68 autor por artigo, considerando que foram publicados 47 artigos e, ainda, que destes, 4 são republicações em periódicos, de artigos já publicados nos anais dos encontros da ANPAD, o que totaliza, em números reais, apenas 43 artigos.

Os dados revelam baixa produção na temática e autores desses artigos não demonstram possuir continuidade de pesquisas nesta área, ou seja, não há autor algum com mais de três trabalhos publicados sobre a temática no período analisado. Dos 79 autores apenas 18 publicaram mais de um artigo no período; os outros publicaram apenas um artigo em todo o período.

Observando-se a distribuição espacial desses autores (Tabela 2), verifica-se que apenas 14 Estados possuem autores que publicaram algum artigo neste período. Considerando que são 27 Estados no Brasil, nos quais há uma quantidade muito grande de órgãos públicos, a concentração em 14 Estados é baixa, o que dificulta a construção de uma comunidade de discurso sobre a temática da cultura e, mais ainda, sobre a cultura em organizações públicas (Carrieri \& Leite-de-Silva, 2006; Fischer \& MacAllister, 2001).

Tabela 2

\section{Produção por Unidade da Federação}

\begin{tabular}{lcc}
\hline \multicolumn{1}{c}{ Estado dos autores } & Quantidade & Percentual \\
\hline Bahia & 1 & 2,00 \\
Ceará & 3 & 6,00 \\
Distrito Federal & 4 & 8,00 \\
Espírito Santo & 1 & 2,00 \\
Goiás & 2 & 4,00 \\
Minas Gerais & 6 & 12,00 \\
Paraíba & 4 & 8,00 \\
Pernambuco & 1 & 2,00 \\
Paraná & 6 & 12,00 \\
Rio de Janeiro & 11 & 22,00 \\
Rio Grande do Norte & 1 & 2,00 \\
\hline
\end{tabular}

Continua 
Tabela 2 (continuação)

\begin{tabular}{lcc}
\hline \multicolumn{1}{c}{ Estado dos autores } & Quantidade & Percentual \\
\hline Rio Grande do Sul & 4 & 8,00 \\
Santa Catarina & 2 & 4,00 \\
São Paulo & 3 & 6,00 \\
York & 1 & 2,00 \\
Total & 50 & 100,00 \\
\hline
\end{tabular}

Nota. Fonte: elaboração dos autores

A maior produção encontra-se no Estado do Rio de Janeiro (11 artigos) seguido dos Estados do Paraná e Minas Gerais com 6 artigos cada um. Não é encontrada produção alguma nos Estados da região norte (Tabela 3). Pesquisas semelhantes realizadas sobre áreas e temáticas diferentes da tratada neste artigo, também apontam a ausência de publicações nas regiões norte e nordeste do Brasil (Camargos et al 2005; Leal, Oliveira, \& Soluri, 2003).

Tabela 3

Produção por região no Brasil

\begin{tabular}{|c|c|c|}
\hline Região & Quantidade & $\%$ \\
\hline \multicolumn{3}{|l|}{ Norte } \\
\hline Nordeste & 10,00 & 20,00 \\
\hline Sudeste & 21,00 & 42,00 \\
\hline Sul & 12,00 & 24,00 \\
\hline Centro-Oeste & 6,00 & 12,00 \\
\hline Exterior & 1,00 & 2,00 \\
\hline Total & 50,00 & 100,00 \\
\hline
\end{tabular}

Nota. Fonte: elaboração dos autores

O que se depreende desse resultado é que a produção científica nesta temática, publicada nos veículos analisados neste estudo, concentra-se nos Estados da federação que possuem instituições de ensino superior com programas de pós-graduação em administração (mestrados e doutorados) com alguma tradição no ensino e pesquisa em administração pública. Vale notar que o Estado da Bahia, apesar da tradição da UFBA neste campo, apresenta um percentual quase nulo de publicações sobre cultura organizacional no setor público.

Uma análise dos dados da Comissão Permanente de Avaliação do Ensino Superior [CAPES], permite verificar que no Brasil existem cursos de mestrados em administração em apenas 16 Estados e que apenas 10 Estados possuem cursos de doutorado. Além disso, na região norte, onde não foi localizado artigo algum sobre a temática de que se trata aqui, existem apenas 2 cursos de mestrado: um no Estado do Pará, na Universidade da Amazônia [UNAMA] e outro no Estado de Rondônia, na universidade Federal de Rondônia [UNIR].

Na perspectiva da possibilidade da criação de uma comunidade de discurso na área de pesquisa em cultura organizacional em organizações públicas foi analisado o perfil dos autores a partir de informações obtidas nos currículos lattes. Estes dados indicam que esses autores que publicaram trabalhos neste período, em sua maioria absoluta, não são pesquisadores na área pública, não são da área de cultura organizacional, e muito menos da área de cultura organizacional em organizações 
públicas. Desses autores, apenas 18 autores, ou seja, 22,78\% do total de 79 têm publicações na área de organizações públicas, enquanto cerca de $58 \%$ dos 79 autores possuem pesquisas sistemáticas em cultura organizacional. Do total de 79 autores apenas 15, ou seja, 18,99\% possuem simultaneamente pesquisas em cultura organizacional em organizações públicas.

Essas considerações chamam a atenção, pois a cultura organizacional, enquanto construto teórico possui aspectos que devem ser particularizados para cada sociedade e organização. Laraia (2001) adverte sobre a particularidade da lógica de cada cultura, enquanto Sahlins (1997, p. 7) chama a atenção para o fato de que "historicidades diferentes, significam culturas diferentes". Isto sinaliza que a compreensão da lógica da cultura organizacional do setor público passa necessariamente pela compreensão de sua historicidade. Neste sentido, a construção de uma comunidade de discurso de cultura organizacional em organizações públicas demanda maior concentração dos pesquisadores nesta área, no sentido de possibilitar a construção de um campo teórico próprio, que leve em conta as especificidades das organizações públicas. Considerando que esses autores são mestres $(43,04 \%)$, doutores (30,38\%) e pós-doutores (7,59\%), observa-se que a produção científica na área é assistemática e que não conseguiu, ainda, reunir e formar uma rede mais extensa de pesquisadores no país.

\section{c) As Publicações}

Na análise do conteúdo dos artigos publicados foram destacadas as características mais importantes da produção no período, a partir das seguintes categorias: natureza da pesquisa; tipo de pesquisa; descrição e importância da pesquisa; questão de pesquisa e/ou objetivos; descrição metodológica da pesquisa; tipos e formas de coleta de dados; abordagem teórica sobre cultura; apresentação dos resultados das pesquisas.

\section{$\underline{\text { Natureza da Pesquisa }}$}

Verificou-se, aqui, como as pesquisas foram classificadas por seus autores, utilizando-se como base as classificações exploratória, descritiva ou causal ${ }^{(1)}$. Dos 47 artigos analisados, 19,15\% foram classificados como estudos exploratórios; 68,09\% como estudos descritivos e 8,51\% como estudos causais. Em 4,26\% desses estudos, os autores não descreveram qual a natureza da sua pesquisa.

\section{$\underline{\text { Tipo de Pesquisa }}$}

Nesta parte buscou-se identificar como as pesquisas realizadas foram consideradas por seus autores no que se refere à classificação dos estudos como sendo qualitativos, quantitativos ou a conjunção dos dois métodos (Mixed Methods Research) ${ }^{(2)}$. Desse modo, 61,70\% dos trabalhos analisados utilizam o método qualitativo, $21,28 \%$ são de base quantitativa, e $17,02 \%$ foram caracterizadas como utilizando a união de métodos qualitativos e quantitativos.

\section{Descrição e Importância da Pesquisa}

Este tópico avaliou a existência de justificativa, teoria de base, questão de pesquisa e a existência de hipóteses ou pressupostos. Procurou-se identificar se havia uma indicação explicita de justificativa da pesquisa, encontrando-se os seguintes resultados: 40,43\% dos artigos destacaram explicitamente as justificativas da pesquisa, enquanto 59,57\% não as apresentam explicitamente. Percebeu-se, nos artigos, uma tendência em justificar o contexto das organizações, das transformações ocorridas na sociedade, mas sem efetivamente apresentar as justificativas para a realização daquela pesquisa.

Com relação à teoria de base, verificou-se em que medida o artigo apresentava uma discussão com os principais autores que tratam do fenômeno estudado ou se apresentava uma discussão menos aprofundada, na medida em que trazia reduzida revisão de literatura e reduzido número de autores que abordam a temática estudada. Assim, a teoria de base foi classificada em aprofundada, superficial ou ausente ou não relacionada, considerando-se ausente ou não relacionada, aquela descrição de uma teoria que não fosse relevante para a abordagem do fenômeno estudado. Os dados apresentam os seguintes resultados: aprofundada, 61,70\%; superficial 36,17\%; e ausente ou não relacionada, 2,13\%. 


\section{Questão de Pesquisa e/ou Objetivos}

Verificou-se, aqui, se o autor enunciou o problema de pesquisa e se descreveu o seu objetivo geral. Para esta verificação utilizou-se, apenas, esta explicitação mencionada pelos autores. A existência de hipóteses ou pressupostos foi também verificada a partir da explicitação (ou não) por parte dos pesquisadores, encontrando-se os resultados apresentados na Tabela 4.

Tabela 4

\section{Descrição e Importância da Pesquisa}

\begin{tabular}{lccc}
\hline \multicolumn{1}{c}{ Características } & Sim (\%) & Não (\%) & Total \\
\hline Questão de pesquisa/objetivo & 89,36 & 10,64 & 100,00 \\
Hipótese/Pressuposto & 29,79 & 70,21 & 100,00 \\
\hline
\end{tabular}

Nota. Fonte: elaboração dos autores

\section{Descrição Metodológica da Pesquisa}

Este tópico verificou se se tratava de uma pesquisa teórica, de um estudo empírico ou de um estudo de caso, avaliando o seu procedimento metodológico. Verificou também se o estudo se caracterizava como longitudinal ou de corte transversal ${ }^{(3)}$. Avaliou, ainda, a utilização de métodos variados ou apenas um tipo de método de análise. Por fim, considerou também a existência detalhada dos procedimentos metodológicos e se houve descrição de como operacionalizar as variáveis definidas.

Em sua maioria trata-se de artigos que foram elaborados a partir de estudos de caso, que totalizam 93,62\% dos trabalhos realizados. Apenas 4,26\% se configuram como trabalhos teóricos, e 2,13\% se consideraram como estudos empíricos. A maioria dos artigos, 74,47\% são estudos de corte transversal, enquanto apenas $25,53 \%$ se refere a estudos longitudinais. Vale a pena ressaltar a importância deste achado, visto que a cultura organizacional é considerada uma construção social (Berger \& Luckman, 2005) e o resultado de trama histórica (Jaime, 2002). Estudar a cultura requer aprofundamento na história das organizações (Laraia, 2001).

Quanto à utilização de métodos variados ou apenas um tipo de método de análise, a indicação - mix de método - considerou a descrição e utilização de mais de um método na elaboração da pesquisa. A grande maioria dos trabalhos utilizou apenas um método. Isso parece divergir das recomendações para o estudo sobre cultura organizacional; por exemplo, de Fleury (1996) que recomenda a utilização de métodos conjugados, ainda com a possibilidade de triangulação não só de métodos, mas também de instrumentos de coleta das informações.

No que se refere à descrição dos procedimentos metodológicos, a maioria dos trabalhos descreve claramente como a pesquisa foi realizada. No mesmo sentido, e em consequência da própria descrição dos procedimentos metodológicos, na maioria dos trabalhos houve descrição de como as variáveis seriam operacionalizadas. Esses dados podem ser visualizados na tabela 5.

Tabela 5

\section{Descrição dos Procedimentos Metodológicos}

\begin{tabular}{lccc}
\hline \multicolumn{1}{c}{ Tópicos analisados } & Sim & Não & TOTAL \\
\hline Mix de método & 38,30 & 61,70 & 100,00 \\
Descrição dos procedimentos & 68,09 & 31,91 & 100,00 \\
Operação das variáveis & 61,70 & 38,30 & 100,00 \\
\hline
\end{tabular}

Nota. Fonte: elaboração dos autores 


\section{$\underline{\text { Tipos e Formas de Coleta de Dados }}$}

Esta parte do trabalho avaliou os tipos de dados, o tipo de amostra, o tamanho da população, o tamanho da amostra e o nível hierárquico dos indivíduos da amostra. Os dados foram classificados como primários, secundários e ambos. Dados primários são aqueles colhidos pelo próprio pesquisador, especificamente para a pesquisa e não disponíveis em outras fontes. Dados secundários são os já disponíveis e utilizados ou coletados por outros pesquisadores, dos quais o pesquisador faz uso. A classificação em ambos indica que o pesquisador utilizou dados primários e secundários na mesma pesquisa.

A análise destaca que 46,81\% dos dados são primários, 8.51\% são secundários; e 44,68\% das pesquisas utilizaram dados primários e secundários simultaneamente (ambos). De qualquer forma, o percentual de 46,81\% de utilização de dados primários pode ser considerado um indicativo de preferência dos pesquisadores em obter as informações de fontes diretas.

A amostra foi classificada como probabilística, incluindo amostras simples, estratificadas, agrupadas e sistemáticas. Neste grupo foram incluídas todas as amostras provenientes da aleatoriedade, sendo definidas como aquelas em que qualquer membro da população tem a mesma possibilidade de pertencer à amostra. O outro tipo de amostra, a não aleatória, não probabilística, é aquela em que o indivíduo não possui as mesmas chances de ser selecionado para pertencer à amostra.

Os dados indicam que a maioria absoluta das pesquisas utilizou a amostra não probabilística, ou seja, em 91,49\% das pesquisas os informantes selecionados para o estudo o foram por algum grau de conveniência do pesquisador. Apenas 8,51\% das pesquisas utilizaram amostra probabilística.

Estes dados devem ser analisados por associação com outras informações, destacando-se os itens tamanho da população e da amostra. Neste aspecto, duas questões são bastante interessantes. Do total das pesquisas, 27 delas, ou seja, 57,45\% não descreveram o tamanho da população, isto é, o texto descreve o tamanho da amostra sem mencionar o tamanho da população. De outro modo, 13 pesquisas não informaram o tamanho da amostra, ou seja, 27,66\% dos trabalhos não dizem quantos indivíduos da população indicada estão sendo estudados. Esta observação pode ser entendida como aspecto de fragilidade nestes estudos, na medida em que ora não indicam o tamanho da população, ora não indicam o tamanho da amostra, impedindo a verificação da representatividade da amostra analisada.

Outro dado ainda a considerar sobre a amostra diz respeito ao próprio tamanho da amostra, quando ambas são informadas. Alguns casos suscitam análises mais detalhadas, tendo em vista a baixa representatividade das amostras.

Podem ser citados os seguintes exemplos: uma das pesquisas informou uma população de 14.000 funcionários, mas a pesquisa foi realizada com apenas 14 indivíduos; outra pesquisa informou uma população de 4.500 indivíduos e fez a pesquisa com apenas 30 indivíduos. É certo que a quantidade dos informantes necessariamente não define a validade das descobertas realizadas nas pesquisas. Entretanto é importante que a amostra possua uma quantidade de indivíduos que possa representar adequadamente o universo pesquisado e o total da população.

Sobre a forma e os instrumentos utilizados para a coleta de dados verificou-se que $19,15 \%$ das pesquisas foram realizadas com a aplicação de questionários, 44,68\% com a realização de entrevistas, $8,51 \%$ com o levantamento documental e $27,66 \%$ com outras fontes e mais de uma fonte de coleta.

No que se refere ao nível hierárquico dos indivíduos pesquisados, buscou-se verificar se os pesquisados eram apenas gestores, subordinados ou gestores e subordinados simultaneamente. Observa-se concentração muito forte das pesquisas dos gestores, com $41,86 \%$ das pesquisas estudando apenas os gestores, e 37,21\% delas estudando gestores e subordinados conjuntamente. Neste aspecto, as pesquisas parecem refletir uma vertente teórica funcionalista que entende serem os gestores os portadores da cultura organizacional. Nesta corrente destacam-se Deal e Kennedy (2000), Fleury (1996) e Schein (2004). No caso de Schein (2004), a sua percepção é de que os gestores são os verdadeiros responsáveis pela implementação, sustentação e mudança da cultura organizacional. 


\section{$\underline{\text { Abordagem Teórica de Cultura }}$}

Outro aspecto avaliado nestes estudos foi a abordagem teórica utilizada pelos autores. Neste sentido, buscou-se identificar se a pesquisa se enquadrava como funcionalista, a qual concebe a cultura como variável organizacional, manipulável pelos gestores e única para toda a organização (Deal \& Kennedy, 2000; Fleury, 1996; Kotter \& Heskett, 1992; Schein, 2004). Ou se a pesquisa se enquadrava em uma abordagem não funcionalista, que pensa a cultura como metáfora da própria organização, não manipulável, sem unicidade para toda a organização e que habita o imaginário coletivo, sem necessariamente constituir um monolítico dentro da organização (Aktouf, 1994; Meyerson \& Martin, 1987).

Os dados encontrados demonstram que em 51,06\% das pesquisas a abordagem foi funcionalista, enquanto em 48,94\% utilizou uma abordagem não funcionalista. Entretanto, em 82,98\% das pesquisas, a cultura foi pensada como sendo única, o que de certa forma reorienta a discussão para a abordagem funcionalista da cultura.

Esta constatação pode ser reflexo da amostra utilizada. Como em grande parte das pesquisas os entrevistados são gestores, muito provavelmente existirá homogeneidade dos significados culturais. Possivelmente a repetição das pesquisas com outros grupos da mesma organização poderia indicar divergências nas percepções da cultura de cada organização.

Ainda sobre a abordagem teórica, este estudo verificou se as pesquisas analisadas levantaram e analisaram características culturais em processo de transformação, de modo a identificar mudanças culturais nas organizações públicas estudadas. Os dados informam que apenas pequena parte das pesquisas se concentrou em analisar mudança cultural. Não houve investimento no sentido de se analisar processos de mudanças culturais, o que se confirma com o que foi encontrado e destacado neste artigo, acerca da temporalidade das pesquisas analisadas - longitudinais ou de corte transversal. Como 74,47\% dos estudos são de corte transversal, não há foco em análise de mudança cultural. Somente o estudo longitudinal permitiria verificar, no tempo, as mudanças culturais dentro das organizações públicas estudadas. Além disso, outra constatação é o fato de que 63,83\% das pesquisas analisaram mudança cultural por mediação de elementos materiais, os chamados artefatos visíveis (Kotter \& Heskett, 1992; Schein, 2004). Algumas apresentam, por exemplo, como mudança cultural, a implantação de processo de avaliação de desempenho.

Apresentação dos Resultados das Pesquisas

Este tópico apresenta a análise dos seguintes elementos: confrontação com a teoria de base, limitações do estudo, recomendações de pesquisa, recomendações aplicadas, validade externa e validade nomológica. Os dados constam da tabela 6.

Tabela 6

Apresentação dos Resultados

\begin{tabular}{lccc}
\hline \multicolumn{1}{c}{ Itens } & Sim & Não & Total \\
\hline Confrontação com a teoria de base & 31,91 & 68,09 & 100,00 \\
Limitações do estudo & 31,91 & 68,09 & 100,00 \\
Recomendações de pesquisas & 38,30 & 61,70 & 100,00 \\
Recomendações aplicadas & 36,17 & 63,83 & 100,00 \\
Validade externa & 21,28 & 78,72 & 100,00 \\
Validade nomológica & 48,94 & 51,06 & 100,00 \\
\hline
\end{tabular}

Nota. Fonte: Elaboração dos autores 
No item - confrontação com a teoria de base - buscou-se avaliar a retomada dos conceitos teóricos discutidos no referencial teórico, para explicar os dados obtidos na pesquisa empírica. Nos quesitos limitações do estudo, recomendações de pesquisa e recomendações práticas, foi observada apenas a sua existência declarada pelos pesquisadores. O item validade externa caracteriza-se pela observância da possibilidade de aplicação dos resultados da pesquisa em outros contextos e situações diferentes das apresentadas no trabalho. O quesito validade nomológica considera o alinhamento dos resultados obtidos com o modelo teórico proposto, bem como a interpretação destes dados, a partir do mesmo modelo construído a partir das hipóteses definidas anteriormente.

Em primeiro lugar, no que se refere à teoria de base, 68,09\% dos trabalhos não rediscutem os conceitos teóricos apresentados para a análise dos dados. Há, em geral, uma apresentação dos dados, seguida de discussão desses dados, mas os pesquisadores não remetem o leitor aos autores citados e às teorias revisadas anteriormente, para dar suporte a essa discussão de dados. E esta forma de apresentação dos dados acaba revelando-se no quesito validade nomológica, isto porque a retomada da teoria de base permitiria avaliar os dados, a partir do modelo teórico apresentado na parte inicial do texto. Vê-se que apenas 48,94\% dos trabalhos apresentam validade nomológica, isto é, retomam o modelo teórico e avaliam os dados, a partir do modelo teórico e das hipóteses construídos inicialmente.

Outro ponto que chama a atenção nesses artigos é a ausência de indicação das limitações do estudo. Tratando-se de temática tão complexa como é a cultura organizacional, que se torna ainda mais complexa quando analisada no âmbito das organizações públicas que são permeadas por relações de poder muito fortes, além de apresentarem outras especificidades, seria quase impossível não haver fatores limitantes, tanto para a realização do estudo, quanto para a sua própria finalização e conclusões. Visto de outra forma, na medida em que a cultura é construção social da realidade (Berger \& Luckmann, 2005), a análise concentrada na pessoa dos gestores, por si só já parece indicar uma limitação do estudo.

No que se refere às recomendações de pesquisa e às recomendações aplicadas ao caso, o percentual também é baixo. Os dados encontrados indicam que não é procedimento normal dos autores destacarem nem as limitações de seus estudos, nem as limitações do método utilizado, nem apresentarem recomendações para o aprofundamento dos resultados obtidos em novos estudos e pesquisas. Este aspecto é semelhante em pesquisas realizadas em outras áreas (Camargos et al, 2005; Sampaio \& Perin, 2006).

\section{CONSIDERAÇÕES FINAIS}

Este trabalho analisou a produção científica brasileira que estuda a cultura organizacional em organizações públicas, considerando a sua relevância tanto do ponto de vista de pesquisa quanto do ponto de vista prático, na medida em que, para o sucesso da reforma do Estado implementada a partir de 1995, foi dada ênfase muito forte à questão da mudança cultural nas organizações públicas. Com esta mudança cultural esperava-se obter dessas organizações a eficiência, eficácia e efetividade no seu desempenho e nos seus resultados.

Foi, assim, estabelecida como hipótese inicial, a existência de vasta produção científica sobre cultura organizacional em organizações públicas, o que não se confirmou, pois a produção de apenas 47 artigos sobre esta temática, no período de 11 anos, no seio da comunidade acadêmica brasileira da área de administração, é muito baixa. Os resultados demonstram que o número de autores que estão envolvidos direta e simultaneamente nesta área de pesquisa, neste período, não passou de 15 . Considerando a importância do tema, isso demonstra pouca sistematicidade e regularidade dos pesquisadores na construção de uma linha de pesquisa de referência e de continuidade para seus trabalhos. 
Outras considerações podem ser feitas a partir dos dados analisados. A produção científica dos pesquisadores brasileiros concentra-se essencialmente em eventos (EnANPAD). Além da baixa produtividade, ainda se percebe uma concentração em poucos Estados brasileiros. Uma região inteira, a região norte, não possui trabalho algum sobre o tema e outras regiões possuem baixíssima produção sobre a temática. Esses resultados sinalizam pouca preocupação acadêmica com uma área de conhecimento que não pode ser desprezada, sobretudo no Brasil, em função do tamanho de sua máquina administrativa e da quantidade de organizações públicas que possui, quer seja na administração direta quer indireta no nível federal, estadual e municipal, o que por si só demandaria um esforço da comunidade científica para a realização de mais pesquisas nesta área.

É, sem dúvida, campo extremamente vasto que demanda novos olhares, de forma a apreender corretamente a realidade das organizações públicas brasileiras, rompendo com o preconceito da suposta imutabilidade da cultura organizacional do setor público e revendo as imprecisões dos conceitos de cultura organizacional no Brasil (Alcadipani \& Crubellate, 2003). Apesar de diversos autores ainda conceberem a cultura brasileira e organizacional como marcada fortemente pelas características de formação do povo brasileiro no ano de 1500, segundo estes autores como a cultura é sujeita a mudanças, é pouco provável que todas as características culturais da formação do Estado brasileiro ainda sejam exatamente as mesmas de 500 anos atrás. Além do mais, a cultura muda, bem como as grandes teorias científicas sofreram transformações nos últimos séculos (Dupuis, 1996). A cultura muda em diversos aspectos, bem como em função de diversos fatores internos e externos, e isto se aplica ao setor público também (Laraia, 2001; Sahlins, 1997). Sendo assim, a mudança cultural nas organizações públicas é passível de ocorrência e demanda efetivamente novos estudos com foco nos processos de transformação cultural.

Outra constatação que emerge deste trabalho refere-se à necessidade de fortalecimento de uma comunidade de pesquisa sobre cultura organizacional em organizações públicas, porquanto, do que se depreende deste estudo, ela ainda não é forte o suficiente para produzir resultados maiores e mais consistentes com a realidade das organizações públicas. Há necessidade de constituição de redes mais amplas dentro da comunidade científica como forma de apreender, de maneira mais adequada, a lógica da cultura organizacional dentro do setor público, o que poderá contribuir com os anseios da sociedade, traduzidos na melhoria da eficiência, eficácia e efetividade do serviço público.

Ainda que este trabalho não se tenha dedicado a análises qualitativas mais aprofundadas acerca dessas pesquisas, questões teóricas, metodológicas, da qualidade dos dados, do seu tratamento e resultados, emergem deste estudo, indicando alguns problemas e fragilidades que são apontados a seguir: a existência de poucos estudos teóricos e de poucos estudos realizados em profundidade; a utilização ampla e generalizada de estudos de caso (ainda que se conheçam as suas limitações); a pouca preocupação com o rigor do método científico, além das limitações do método utilizado na pesquisa; a dificuldade sistemática de articulação do referencial teórico, como o estudo empírico, além da generalização rápida de achados a partir de uma só pesquisa realizada por meio de um único estudo de caso, utilizando amostras limitadas e eventualmente tendenciosas.

As conclusões aqui apresentadas devem ser seguidas das limitações inerentes a trabalhos desta natureza. Neste sentido, a subjetividade dos pesquisadores, sem sombra de dúvida, pode representar interferência na forma de perceber cada um dos tópicos analisados, e afetar a compreensão final dos trabalhos. Outras pesquisas nesta mesma linha podem ser feitas como forma de obtenção de novas informações sobre a produção intelectual acerca da cultura organizacional em organizações públicas.

Esta pesquisa, bem como as realizadas por (Camargos et al., 2005; Leal et al., 2003), citadas neste trabalho, podem ser utilizadas como base para comparações com diversas áreas, para se obter uma análise mais completa da produção científica no Brasil em campos específicos da administração, redirecionando, redefinindo, reestruturando e fortalecendo os campos. Por fim, este trabalho lança um convite à reflexão para a comunidade acadêmica, tanto para aqueles que tratam da cultura organizacional, como para os que militam no campo da administração pública, de modo que se possa construir uma comunidade científica em torno de estudos de cultura organizacional voltada para o setor público.

\section{Artigo recebido em 13.12.2008. Aprovado em 17.06.2009.}




\title{
NOTAS
}

\begin{abstract}
${ }^{1}$ Os estudos exploratórios permitem ao investigador aumentar sua experiência em torno de determinado problema. Ele parte de uma hipótese e aprofunda seu estudo nos limites da realidade específica, buscando antecedentes e maior conhecimento para, em seguida, planejar uma pesquisa descritiva ou do tipo experimental (Trivinos, A. N. S. (1995). Introdução à pesquisa em ciências sociais: a pesquisa qualitativa em educação (p. 109). São Paulo: Atlas.). Os estudos descritivos pretendem definir com exatidão os fatos e fenômenos de determinada realidade (Trivinos, 1995). Eles têm como objetivo informar o pesquisador sobre situações, fatos, opiniões ou comportamentos da população analisada, buscando mapear a distribuição de um fenômeno na população estudada (Sampaio \& Perin, 2006). Os estudos causais são aqueles que testam uma teoria e suas relações causais (Sampaio \& Perin, 2006).

2 Os estudos qualitativos objetivaram uma interpretação mais ampla do fenômeno social em estudo, visando à sua compreensão. O significado é a preocupação essencial na abordagem qualitativa (Trivinos, 1995). Os estudos quantitativos consideram elementos básicos, como o fornecimento de descrições quantitativas da população estudada, a realização de coleta de dados por meio de instrumentos estruturados e o uso de informações coletadas numa amostra da população analisada (Sampaio \& Perin, 2006). A terceira classificação mostra a opção do pesquisador em utilizar os dois métodos simultaneamente na busca do fortalecimento dos argumentos utilizados.
\end{abstract}

${ }^{3}$ Considera-se estudo longitudinal o que analisa o fenômeno ao longo de um tempo definido, e transversal aquele que analisa o fenômeno em dado momento.

\section{REFERÊNCIAS BIBLIOGRÁFICAS}

Aktouf, O. (1994). O simbolismo e a cultura de empresa: dos abusos conceituais às lições empíricas. In J. F. Chanlat (Coord.), O indivíduo na organização: dimensões esquecidas (pp. 40-79). São Paulo: Atlas.

Alcadipani, R., \& Crubellate, J. M. (2003). Cultura organizacional: generalizações improváveis e conceituações imprecisas. Revista de Administração de Empresas, 43(2), 64-77.

Berger, P., \& Luckmann, T. (2005). A construção social da realidade: tratado de sociologia do conhecimento (25a ed.). Petrópolis: Vozes.

Bresser Pereira, L. C., \& Spink, P. (1998). Reforma do Estado e administração pública gerencial. Rio de Janeiro: Editora FGV.

Camargos, M. A., Coutinho, E. S., \& Amaral, H. F. (2005, setembro). O perfil da área de finanças do EnANPAD: um levantamento da produção científica e de suas tendências entre 2000-2004. Anais do Encontro Nacional da Associação Nacional de Pós-Graduação e Pesquisa em Administração, Brasília, DF, Brasil, 29.

Carrieri, A. P., \& Leite-de-Silva, A. R. (2006). Cultura organizacional versus cultura nas organizações: conceitos contraditórios entre o controle e a compreensão. In M. Marchori (Org.), Faces da cultura e da comunicação organizacional (pp. 51-75). São Caetano do Sul: Difusão Editora.

Cavedon, N. R., \& Fachin, R. C. (2002). Homogeneidade versus heterogeneidade cultural: um estudo em uma universidade pública. Organizações \& Sociedade, 9(25), 61-76.

Deal, T. E., \& Kennedy, A., (2000). Corporate cultures. The rites and rituals of corporate life. Cambridge: Basic Books.

Decreto-Lei n. 200 de 25 de fevereiro de 1967 (1967). Dispõe sobre a organização da Administração Federal, estabelece diretrizes para a Reforma Administrativa e dá outras providências. Brasília, 
DF. Recuperado em 20 de novembro de 2009, de http://www.planalto.gov.br/ccivil/decretolei/del0200htm

Dupuis, J. P. (1996) Antropologia, cultura e organizações: proposta de um modelo construtivista. In J. F. Chanlat (Org.), O indivíduo na organização: dimensões esquecidas (Vol III, pp. 231-251). São Paulo: Atlas.

Fadul. E., \& Souza, A. R. (2005, setembro). Políticas de reformas da administração pública brasileira: uma compreensão a partir de seus mapas conceituais Anais do Encontro Nacional da Associação Nacional de Pós-Graduação e Pesquisa em Administração, Brasília, DF, Brasil, 29.

Fischer, T., \& Mac-Allister, M. (2001). Nota técnica: jogando com a cultura organizacional. In S. R. Clegg, C. Hardy, \& W. R. Nord (Eds.), Handbook de estudos organizacionais: reflexões e novas direções (Vol. 2, Chap 12, pp. 252-259). São Paulo: Atlas.

Fleury, M. T. L. (1996). O desvendar a cultura de uma organização: uma discussão metodológica. In M. T. L. Fleury \& R. M. Fischer (Orgs.), Cultura e Poder nas organizações (2a ed., pp. 15-27). São Paulo: Atlas.

Freitas, M. E. (1991). Cultura organizacional: grandes temas em debate. Revista de Administração de Empresas, 31(3), 73-82.

Freitas, M. E. (2007). Cultura organizacional: evolução e crítica. São Paulo: Thomson Learning.

Hofstede, G. (2003). Culturas e organizações. Compreender a nossa programação mental (2a ed.). Lisboa: Edições Silabo.

Jaime, P., Jr. (2002). Um texto, múltiplas interpretações: antropologia hermenêutica e cultura organizacional. Revista de Administração de Empresas, 42(4), 72-83.

Jaime, P., Jr., \& Serva, M. (1995). Observação participante e pesquisa em administração: uma postura antropológica. Revista de Administração de Empresas, 3(1), 64-79.

Kotter, J. P., \& Heskett, J. L. (1992). Corporate culture and performance. New York: The Free Press.

Laraia, R. B. (2001). Cultura: um conceito antropológico (14a ed.). Rio de Janeiro: Jorge Zahar.

Leal, R. P. C., Oliveira, J., \& Soluri, A. F. (2003). Perfil da pesquisa em finanças no Brasil. Revista de Administração de Empresas, 43(1), 91-104.

Loureiro, M. R., Abrúcio, F., \& Rosa, C. A. (1998). Radiografia da alta burocracia federal brasileira. Revista do Serviço Público, Ano 49 (4), 47-84.

Marini, C. M. F. (1996). Crise e reforma do Estado: uma questão de cidadania e valorização do Servidor. Revista do Servidor Público, 47(3), 5-33.

Martin, J., \& Frost, P. (2001). Jogos de guerra da cultura organizacional: a luta pelo domínio intelectual. In S. Clegg, C. Hardy, \& W. R. Nord (Eds.), Handbook de estudos organizacionais: reflexões e novas direções (Vol. 2, Chap 11, pp. 219-251). São Paulo: Atlas.

Mascarenhas, A. O. (2007, setembro). Desenvolvimento, mudança cultural e a formação do comportamento empreendedor - um estudo de caso. Anais do Encontro Nacional da Associação Nacional de Pós-Graduação e Pesquisa em Administração, Rio de Janeiro, RJ, Brasil, 31.

Meyerson, D., \& Martin, J. (1987). Cultural change: an integration of three different views. Journal of Management Studies, 24(6), 623-647.

Ministério da Administração Federal e da Reforma do Estado. (1995). Plano diretor da Reforma do Aparelho do Estado. Brasília: Imprensa Nacional. 
A Produção Científica Sobre Cultura Organizacional em Organizações Públicas no Período de 1997 a 2007: 669 Um Convite à Reflexão

Pires, J. C. S., \& Macêdo, K. B.(2006). Cultura organizacional em organizações públicas no Brasil. Revista de Administração Pública, 40(1), 81-105.

Sahlins, M. (1985). Cultura e razão prática. Rio de Janeiro: Zahar.

Sahlins, M. (1997). Ilhas de história. Rio de Janeiro: Jorge Zahar Editor.

Sampaio, C. H., \& Perin, M. G. (2006). Pesquisa científica da área de marketing: uma revisão histórica. Revista de Administração Contemporânea, 10(2), 179-202.

Schein, E. (2004). Organizational culture and leadership (3a ed.). San Francisco: Jossey-Bass.

Smircich, L. (1983). Concepts of culture and organizational analysis. Administrative Science Quarterly, 28(3), 339-358. 